\title{
Efficient Two-Step Approach for Automatic Number Plate Detection
}

\author{
Ievgen Gorovyi
}

\begin{abstract}
-intelligent transportation systems are rapidly growing mainly due to active development of novel hardware and software solutions. In the paper a problem of automatical number plate detection is considered. An efficient two-step approach based on plate candidates extraction with further classification by neural network is proposed. Stroke width transform and contours detection techniques are utilized for the image preprocessing and extraction of regions of interest. Different local feature sets are used for the final number plate detection step. Efficiency of the developed method is tested with real datasets.
\end{abstract}

Keywords-automatic number plate recognition (ANPR), stroke width transform, features extraction, neural network

\section{INTRODUCTION}

I NTELLIGENT transportation systems have a significant impact on a productivity and security of humanity. With development of advanced signal and image processing solutions applications of various systems are rapidly growing. This includes various parking systems, automatic traffic control, traffic law enforcement, vehicle detection and tracking [1] and many more. Incorporation of modern computer vision and machine learning techniques opens new perspectives in such sophisticated applications as road sign recognition [2] and traffic flow prediction [3]. Given practical examples indicate on a high potential of computational methods and artificial intelligence in global transportation and its efficiency.

The automatical number plate recognition (ANPR) systems have a special place in this consideration.

A typical ANPR system is based on three important steps: number plate detection, segmentation and character recognition (Fig. 1). At first, the image region corresponding to the number plate should be carefully located. This is a crucial step of ANPR system affecting the overall performance. After that the detected plate area is analyzed in order to automatically separate the text symbols. Finally, a proper optical character recognition engine (OCR) is applied for the recognition step [4].

The challenge is that many different imaging scenarios exist in real conditions. In particular, changing illumination level, nonstationary background and vision geometry. Obviously that the number plates could have different sizes, locations in the frame, colors and font styles. The existence of plate skewness also significantly complicates the detection step.

This paper is an extended version of the Signal Processing Symposium paper presented in Signal Processing Symposium, Debe, Poland, June 10 $12,2015$.

Ievgen Gorovyi is with the Institute of Radio Astronomy, Kharkiv, Ukraine, (e-mail: gorovoy@rian.kharkov.ua).
In order to perform the number plate detection in efficient way, various local image features can be utilized [4]. For example, the analysis of image edges can be used for this purpose [4]-[5]. In [6] authors demonstrated the potential of color edges analysis. Specific image preprocessing procedure based on the local contrast enhancement is proposed in [7]. It is illustrated how the information about the vertical edges can be used for the successful detection of the number plate area. Also it was shown that the Hough transform can be applied for the number plate detection problem [8]. However, this approach is time-consuming and can not be used in real-time. Connected component analysis (CCA) is also a popular tool for the analysis of binary images. According to this approach, the binary image is scanned and the pixel connectivity is analyzed. Based on special properties of the plate area regions, the number plate can be extracted from the image [9]. More detailed review of the state-of-the art number plate detection methods can be found in [4].

In the paper a novel two-step approach for the automatic number plate detection is proposed. The first step is based on the plate candidates areas extraction. This is achieved via application of the stroke width transform (SWT) [10] together with contour detection method. The second step is accomplished by means of the neural network (NN) which classifies the obtained sequence of number plate candidates.

In Section II important image preprocessing steps required for the successful plate candidates extraction are described. The key principles of SWT are considered. The specific alternative contour detection scheme is introduced as well. In Section III the main ideas related with the feature extraction

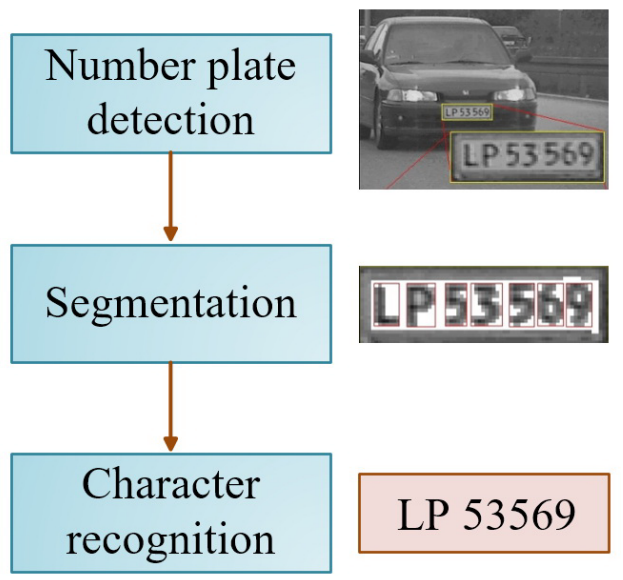

Fig. 1. Main steps ANPR system 
for the classifier training are outlined. Experimental results of $\mathrm{NN}$ testing are illustrated and discussed.

\section{Extraction of Number Plate Candidates}

In this section, key steps for the extraction of number plate candidates are described. In particular, important image preprocessing steps, text regions detection with stroke width operator and blob extraction procedure.

\section{A. Text Detection and Stroke Width Transform}

The text detection in natural scenes is a popular and challenging problem [11]. Areas of application include industrial automation, multimedia retrieval, signs recognition and others. Uneven illumination, complex backgrounds and various text layouts make the detection problem quite challenging. In the paper we consider such problem within the scope of the number plate detection since its area contains the text.

In order to locate the image regions containing the text, we have utilized the SWT. This methodology can be considered as the application of a specific local image operator. It is based on the calculation of special metrics for each image pixel.

In order to perform required analysis, at first the pair of Sobel gradients is calculated

$$
G_{x}^{\text {Sobel }}=\left[\begin{array}{ccc}
-1 & 0 & 1 \\
-2 & 0 & 2 \\
-1 & 0 & 1
\end{array}\right], G_{y}^{\text {Sobel }}=\left[\begin{array}{ccc}
-1 & -2 & -1 \\
0 & 0 & 0 \\
1 & 2 & 1
\end{array}\right] .
$$

Here $G_{x}^{\text {Sobel }}, G_{y}^{\text {Sobel }}$ are horizontal and vertical gradient kernels. The gradient images are evaluated as a convolution of the input image with the given kernels. Thus, the total intensity gradient and its direction can be calculated as

$$
G_{I}^{\text {Sobel }}=\sqrt{\left(G_{x}^{\text {Sobel }}\right)^{2}+\left(G_{y}^{\text {Sobel }}\right)^{2}}, \quad \varphi=\frac{G_{y}^{\text {Sobel }}}{G_{x}^{\text {Sobel }}} .
$$

Fig. 2 illustrates an example of calculated Sobel gradients of the image containing the number plate. One can observe that the gradient values are close to zero in smooth image regions and demonstrate the significant change at borders. In particular, the number plate area is clearly seen. Some methods based on vertical and horizontal image gradients changing are utilized for the plate detection area [4]. The final step before the construction of SWT image is calculation of the edge map. A good choice is usage of Canny edge detector [12] for this purpose. Fig. 3(a) contains an input image. An example of calculated gradient directions is shown in Fig. 3(b). Obtained edge map is shown in Fig. 3(c).

One can see that the number plate borders are presented in the edge map. After calculation of gradient directions (Fig. 3(b)) and image edge map (Fig. 3(c)) we are ready to apply the SWT operator. The basic idea is illustrated in Fig. 4(a). We follow the ray $r=p+n d_{p}$, where $d_{p}$ is gradient direction at the pixel $p$, until the edge pixel $q$ is not found (Fig. 4)

If the $d_{p}$ is roughly opposite, each pixel of output image with stroke widths along the direction along the segment $[p, q]$ is assigned to the width $|p q|$. As a result one can obtain the
SWT image (Fig. 4(b)). Introduced operator has a local nature since the stroke width is calculated for each image pixel. One can observe the areas of equal stroke width in Fig. 4(b). The next task is to combine such areas which will result in the list of the letter candidates. In order to do this several filtering rules are developed. At first, the pixels with equal stroke widths (stroke width ratio threshold is used) are clustered into the letter candidates. After that the letters can be extracted from the full list of connected components based on stroke width variances within each connected component, the limitation of the aspect ratios and maximum acceptable height of the component. As a result, the letters are detected.

The next step of the text detection algorithm is combining of the letters into the words. The logic is straightworward: letters with similar spacing, orientation and color are combined. Additional filtering is performed with respect to the stroke width and letter sizes. Example of words detection is illustrated in Fig. 5.

Green rectangles correspond to the extracted letters and the final red rectangle contains the number plate area. One can see that it is significantly skewed, however applied methodology can successfully handle such geometry.

\section{B. Blobs Extraction Procedure}

In order to increase the final detection rate, we propose to use an alternative scheme for the location of the number plate area.

It is known that one of the options for the number plate detection is application of CCA for the binary image. Before performing the binarization itself, we propose to apply several image preprocessing steps. At first, the initial smoothing is applied. Good options are median blur or conventional Gaussian filter. After that the morphological gradient is calculated. The morphological gradient is determined as a difference between the eroded and dilated images [13]. This operator has it highest values when the image intensity is changing most rapidly [13]. In particular, such areas correspond to the number plate borders.

An example of calculated morphological gradient is shown in Fig. 6(a). The convolution of this image with a uniform box filer allows to increase the contrast (Fig. 6(b))

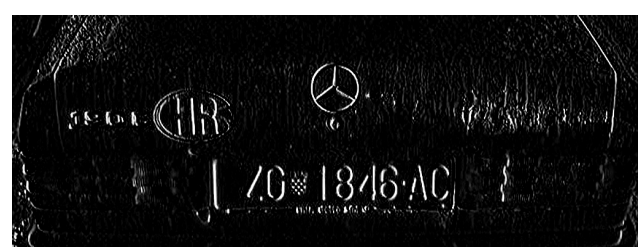

(a)

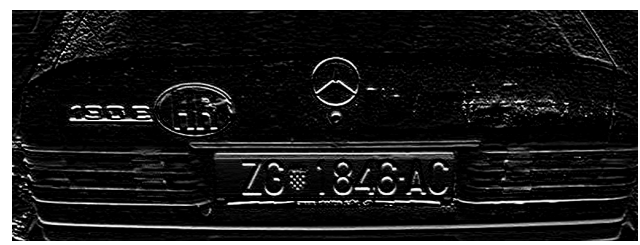

(b)

Fig. 2. Sobel gradients example 
For the image binarization several options are available. A common global binarization based on a single threshold value

$$
I_{\text {binary }}=\left\{\begin{array}{l}
1, I \geq I_{t h} \\
0, I<I_{t h}
\end{array}\right.
$$

where $I_{t h}$ is a constant threshold applied for a whole image. However such global thresholding can be often inefficient due to uneven illumination and some shadows in the camera frame. A good alternative is the adaptive thresholding, where separate thresholds are applied in sliding windows [13]. After the image binarization, the conventional contour detector [14] is applied. Obtained contours can be filtered with respect to the area and the aspect ratio of the corresponding contour bounding rectangles.

An example of extracted blobs (filled contours) and corresponding bounding rectangles in the input image is given in Fig. 7. The detected number plate candidates based on the developed alternative scheme are saved for the further analysis.

After the separate application of SWT for the text detection and described blob extraction algorithm, the final number plate candidates are found. The block-scheme in Fig. 8 contains the main steps of the described procedures. After the separate application of SWT for the text detection and described blob extraction algorithm, the final number plate candidates are found.

The crucial point for the ANPR system is low false negative rate. In other words, it is better to detect more number plate candidates than to skip some true plate areas in some images. Described hybrid procedure for candidates extraction successfully fulfills such requirements.

An important step is to properly classify the obtained number plate candidates. Important findings are described in the next section.

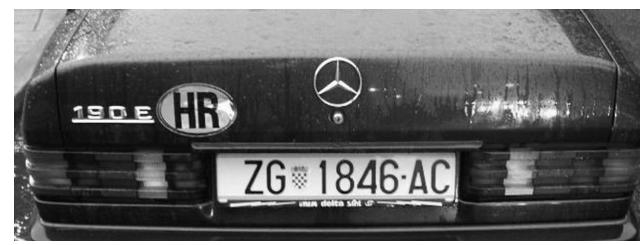

(a)

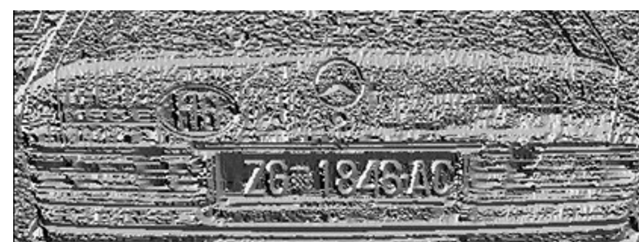

(b)

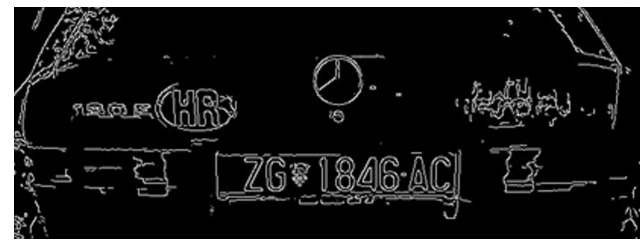

(c)

Fig. 3. Gradients calculation ( $\mathrm{a}-$ input image, $\mathrm{b}$ - gradient directions, $\mathrm{c}-$ Canne edge map)

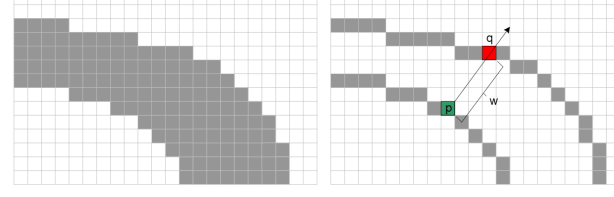

(a)

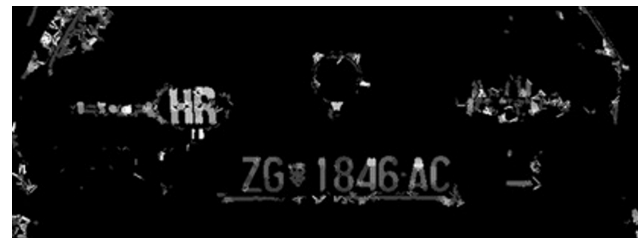

(b)

Fig. 4. Stroke width calculation ( $\mathrm{a}-\mathrm{SWT}$ principle, $\mathrm{b}-\mathrm{SWT}$ image)

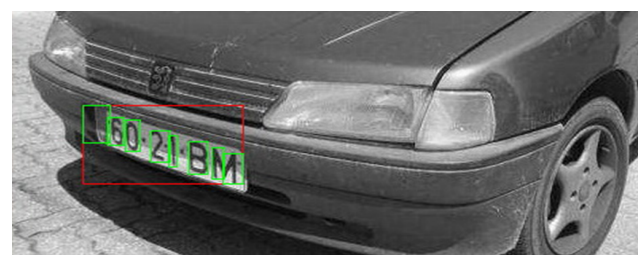

Fig. 5. Example of text detection with SWT

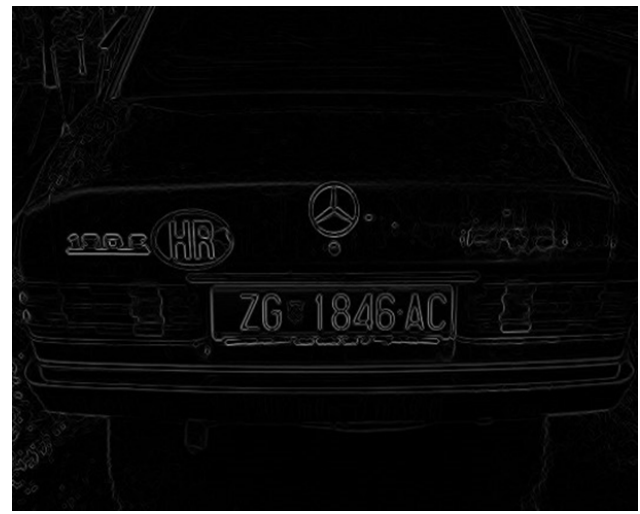

(a)

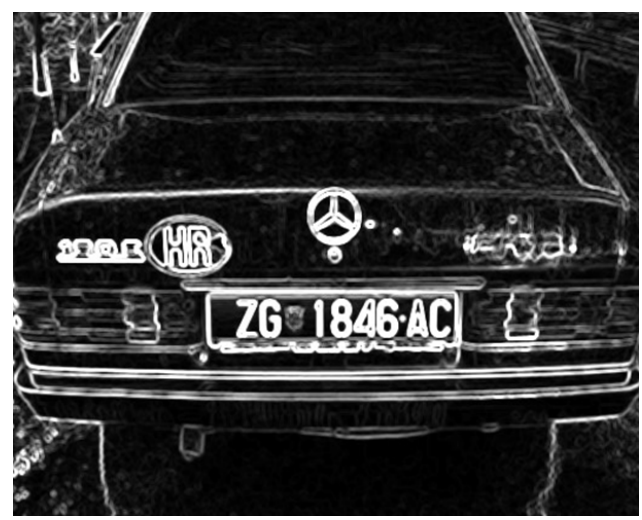

(b)

Fig. 6. Application of convolutional operator (a - morphological gradient, $\mathrm{b}-$ contrast enhancement)

\section{ClaASSIFICATION EXPERIMENT}

In this section the features extraction step and classification experiment are described. 


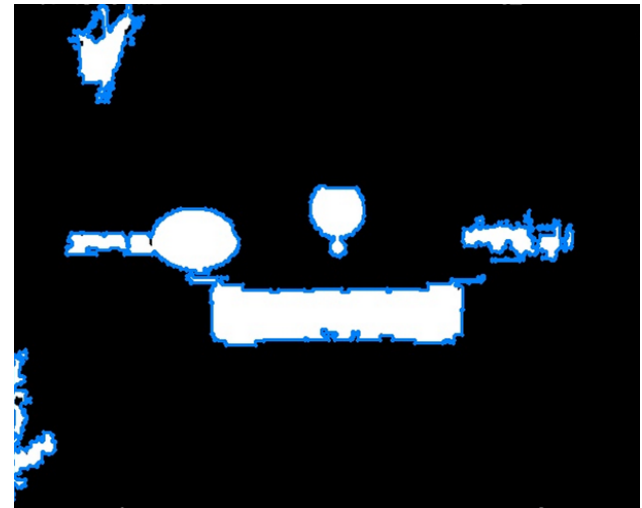

(a)

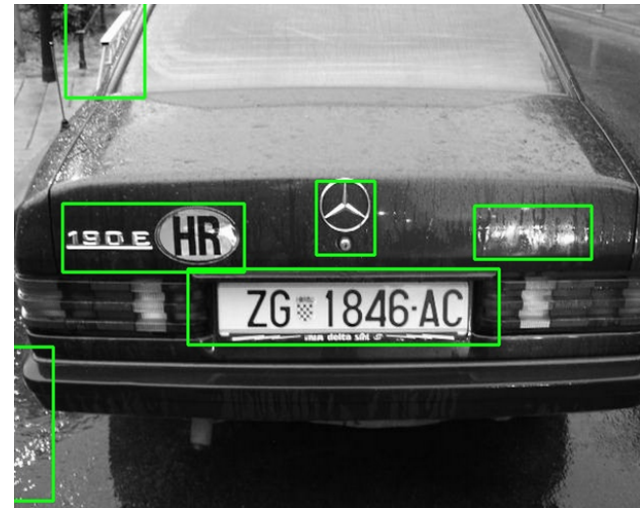

(b)

Fig. 7. Ilustration of alternative preprocessing scheme

\section{A. Features Extraction and Input Dataset}

Described hybrid approach returns the list of the number plate candidates. The last and the most important step is to determine the true plate image among the extracted list Prepared training dataset of vehicle images is used for the evaluation of the number plate candidates. Fig. 9 illustrates the part of arbitrary taken images from the training dataset. The training dataset contains vehicle images with different aspect rations, various skewness level and imaging geometry. For the classification of plate candidates we have trained the 3-layer perceptron NN. An important step for the classification experiment is features extraction. We have utilized 3 types of features: statistical features, Haar-based features and features extracted by the principal component analysis (PCA) [15]. For statistical features first three statistical moments are utilized (mean, variance, skewness)

$$
\begin{gathered}
\mu=\frac{1}{N_{X} N_{Y}} \sum_{i} \sum_{j} I(i, j), \\
\sigma^{2}=\frac{1}{N_{X}, N_{Y}}=\sum_{i} \sum_{j}|I(i, j)-\mu|, \\
\gamma=\frac{1}{N_{X} N_{Y}} \frac{\sum_{i} \sum_{j}|I(i, j)-\mu|^{3}}{\sigma^{3 / 2}},
\end{gathered}
$$

where $I(i, j)$ is image pixel. Fig. 10 contains an example of constructed histograms for the true number plate area and random number plate candidate. One can see that the

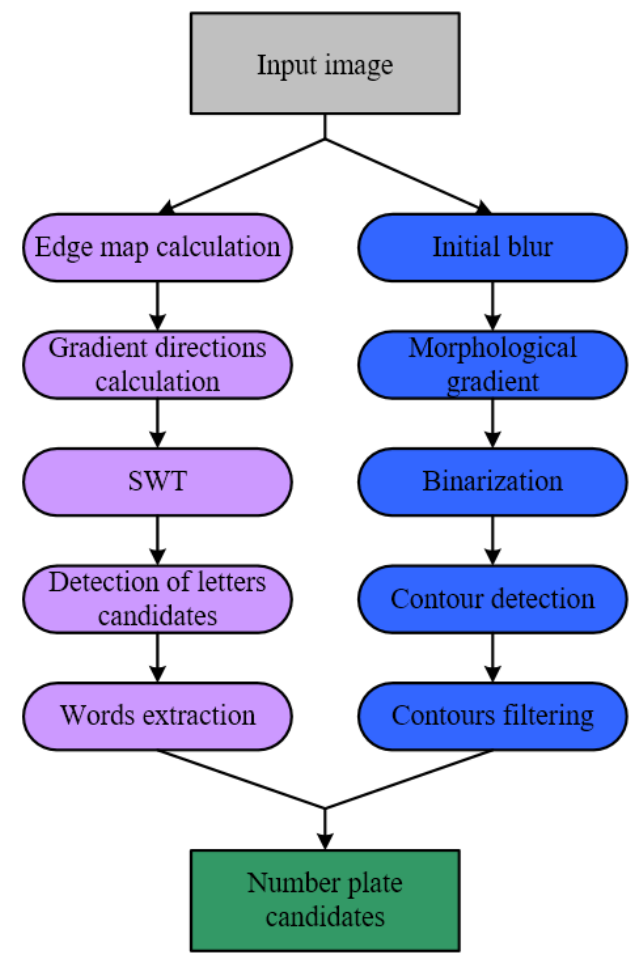

Fig. 8. Block-scheme of the developed detection algorithm

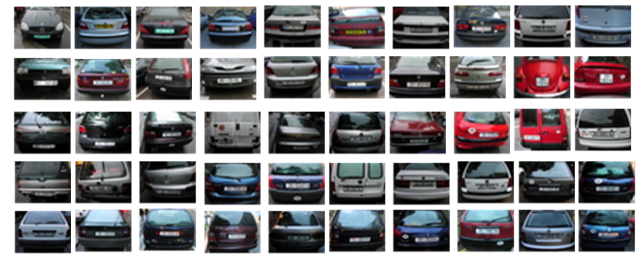

Fig. 9. Example of part of training dataset

pixel intensities distribution are quite distinguishable which indicates on suitability of statistical features. In particular, the most of background of the true number plate is smooth and uniform. The plate characters correspond to the peak in histogram (for black text this peak appears at low intensities).

Haar-based image features [16] are efficient tool often used for the face detection. The most efficient wat to calculate such features is to construct the so-called integral image. It is determined as

$$
I_{I}(i, j)=\sum_{i^{\prime}=1}^{i} \sum_{j^{\prime}=1}^{j} I\left(i^{\prime}, j^{\prime}\right),
$$

where each pixel of the integral image is equal to a sum within the rectangular area of the input image. Since the Haarbased features are defined as a partial sums within rectangular patches, feature extraction is perfomed in efficient way using (4).

Another option for the features extraction is to treat the whole images as features. This can be done using the principal component analysis (PCA). At the first step of PCA each number plate candidate image is considered as a high-dimension data point. Based on the existing training set of images, the 


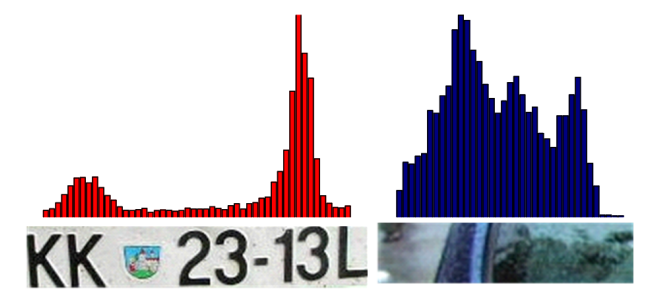

Fig. 10. Calculated histograms for true number plate and arbitrary candidate

input feature matrix is considered

$$
X_{i j}, \quad i=1 \ldots N, \quad j=1 \ldots N d,
$$

where $N$ is the number of images in the training dataset, $N d$ is the length of the column-vectorized images. According to the PCA, the data matrix is transformed to another basis, which contains so-called principal components. The variance of such components corresponds to the eigenvalues of the sample covariance matrix [15]. The number of extracted feature corresponds to the number of retained principal components. It is often the most of the variance is concentrated in only few PCs and this is used for the data compression. After such manipulations the extracted feature matrix is used as input for the NN.

\section{B. Training and Testing of Neural Network}

After feature extraction step we performed the training of the classifier. This is typically considered as the estimation of NN structure having input feature matrix and labeled outputs (classes) [17]. In our problem the goal is to automatically determine whether the input image patch is the number plate or not. Fig. 11 contains the architecture of utilized 3-later perceptron NN. Such 3-layer model is quite simple but at the same time it is possible to perform the nonlinear separation of the data. The NN represents a directed graph where each of layers is fully connected to the next one. For the given input feature vector the weighted sum of features is calculated for a set of hidden layer nodes

$$
y_{j}=f\left(\sum_{i} w_{1 i} x_{j i}+b_{1}\right),
$$

where $w_{1 i}$ corresponds to the NN weights, $b_{1}$ is bias, $f$ is nonlinear activation function [17]. Consequently, the outputs

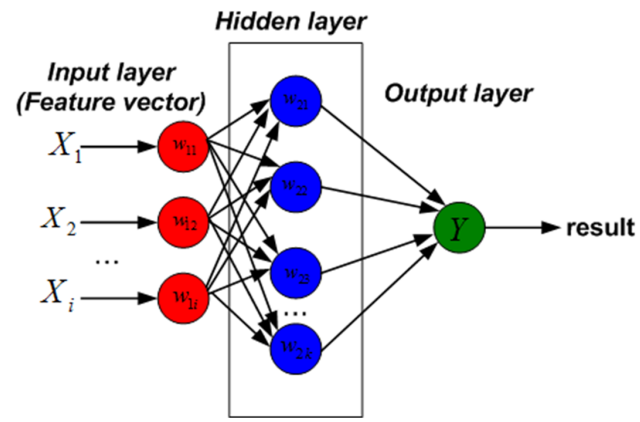

Fig. 11. Structure of used NN of the hidden layer are used as inputs for the 3rd NN layer. The final result is compared with predefined threshold in the following way

$$
Y=\left\{\begin{array}{l}
1, \text { output } \geq \text { th } \\
0, \text { output }<\text { th }
\end{array} .\right.
$$

All parameters of $\mathrm{NN}$ are estimated at the training stage using a conventional backpropagation method [17].

After training of the classifier the system is ready for the analysis of new test data. The feature extraction step is applied for the detected number plate candidates from the testing dataset. Trained NN returns the label for each of extracted plate candidates.

One of the most commonly used instruments for the description of classification performance is receiver operating characteristics (ROC). It represents the dependence of true positive rate (TP) on the false positive rate (FP) [18]. Fig. 12 illustrates the ROC curve obtained for 3 different learning scenarios. One can see that the usage of PCA features leads to better classification accuracy than for the introduced local features. The best classification results are obtained for the case of usage of combined features set. Given experimental results indicate on a high performance of the developed hybrid procedure for the automatical number plate detection.

This paper is an extended version of the publication from signal processing symposium (SPSympo-2015) [19].

\section{CONCLUSION}

In the paper, the algorithm for the automatic number plate detection is proposed. The text extraction algorithm based on the stroke width transform is described. In order to improve the detection rate, an alternative plate candidates extraction procedure based is developed. It is based on the morphological analysis and contours detection. As a result, more efficient detection of the number plate candidates is performed. The final number plate location is accomplished by means of neural network trained on different feature sets. The developed method successfully handles different imaging scenarios, uneven lighting conditions and can be easily integrated into various intelligent transportation systems.

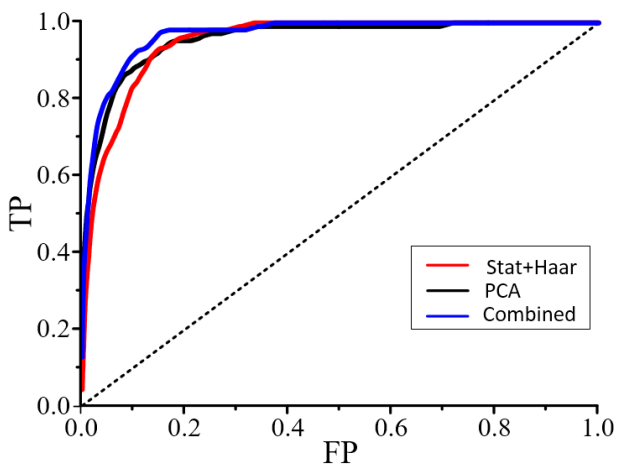

Fig. 12. ROC curves for different feature sets. 


\section{REFERENCES}

[1] A. Mukhtar, A. L. Xia, T. B. Tang, Vehicle Detection Techniques for Collision Avoidance Systems: A Review, IEEE Transactions on Intelligent Transportation Systems, Vol.16 ,No.5,2015, pp. 2318-2338.

[2] J. Greenhalgh and M. Mirmehdi, Recognizing Text-Based Traffic Signs, IEEE Transactions on Intelligent Transportation Systems, Vol.16 ,No.3, 2015, pp. 1360-1369.

[3] L. Yisheng et al, Traffic Flow Prediction With Big Data: A Deep Learning Approach, IEEE Transactions on Intelligent Transportation Systems, Vol.16 ,No.2,2015, pp. 865-873.

[4] S. Du, M. Ibrahim, M. Shehata and W. Badawy, Automatic License Plate Recognition (ANPR): A state of the art review, IEEE Trans. On Circuits and Systems for Video Technology, Vol. 23, Issue 2, pp.311-325, 2013.

[5] H. Bai and C.Liu, A hybrid license plate extraction method based on edge statistics and morphology, Int. Conf. Patt. Recog., Vol. 2, pp. 831834, 2004.

[6] S. L. Chang, L. S. Chen, Y. C. Chung and S. W. Chen, Automatic license plate recognition, IEEE Trans. On Intelligent Transportation Systems, Vol. 5, No. 1, 2004

[7] D. Zheng, D. Zhao, J. Wang, An efficient method of license plate location, Pattern Recognition Letters, Vol. 26, 2005, pp. 2431-2438.

[8] V. Kamat and S. Ganesan, An efficient implementation of the Hough transform for detecting vehicle license plates using DSPs, Real-Time Tech. and App. Symp, pp. 58-59, 1995.

[9] $\mathrm{P}$. Wu et al, License plate extraction in low resolution videos, Pattern Recog., Vol. 1, pp. 824-827, 2006.
[10] B. Epshtein, Y. Wexler and E. Ofek, Detecting Text in Natural Scenes with Stroke Width Transform, IEEE International Conference on Computer Vision and Pattern Recognition, 2010, pp.2963 2970.

[11] Q. Ye and D. Doermann, Text detection and recognition in imagery: A survey, IEEE Trans. on Pattern Analysis and Machine Intelligence, Vol. 37, No. 7, 2015, pp. 1480-1500.

[12] J. Canny, A computational approach to edge detection, IEEE Trans. on Pattern Analysis and Machine Intelligence, Vol. 8, No. 6, pp. 679-698, 1986.

[13] G. Bradsky and A. Kaehler, Learning OpenCV, OReally Media Inc., 2008.

[14] S. Suzuki and K. Abe, Topological structural analysis of digitized binary images by border following, Computer vision, graphics and image processing, Vol. 30, pp. 32-46, 1985.

[15] I. T. Jolliffe, Principal Component Analysis, 2n edition, Springer, 2002

[16] P. Viola and M. Jones, Rapid object detection using a boosted cascade of simple features, IEEE International Conference on Computer Vision and Pattern Recognition, pp. 511-518, 2001.

[17] S. Haykin, Neural networks: A Comprehensive Foundation, 2nd edition, Pearson Prentice Hall, 823p., 1999.

[18] T. Fawcett, An introduction to ROC analysis, Pattern Recognition Letters, Vol. 27, pp. 861-874, 2006

[19] I. Gorovyi and I. Smirnow, Robust number plate detector based on stroke width transform and neural network, Proceedings of Signal Processing Symposium (SPSympo-2015), Debe, Poland, 2015, pp.163-166. 